\title{
DESIGN OF THE MODERN PASTURE BATCHING SYSTEM BASED ON WEIGHING INSTRUMENT
}

\author{
Hu Jie ${ }^{1}$, Zhou Zhifeng ${ }^{2}$, Chen Wenlong ${ }^{3}$ \\ ${ }^{1}$ Postgraduate, College of Mechanical Engineering, Shanghai University of Engineering Science, Shanghai, China \\ ${ }^{2}$ Associate Professor, College of Mechanical Engineering, Shanghai University of Engineering Science, Shanghai, \\ China \\ ${ }^{3}$ Changzhou WeiBo weighing equipment system Co, Ltd. Jiangsu Changzhou, China
}

\begin{abstract}
For dairy cattle total mixed ration (TMR) feeding technology is the inevitable requirement of modern ranch, It was proposed that a ranch batching system based on weighing instrument, to achieve the production process of dairy cattle feed ingredients section of the fully automated production. Depend on the requirement of field process, the weighing instrument called ID510 is the ingredients and control core for the system, to realize the realization automated weighing ingredients for production equipment; write the PC code by using C++ Builder 6.0 to go through the human-computer interaction that used serial communication between weighing instrument and the upper computer. It could display the ingredient data by using Ethernet communication by UDP (user datagram protocol) between PC and screen monitor. It could realize management of recipes, ingredient monitoring, statistical report forms, historical data inquiry function by compiling ACCESS database. In actual batching process, the batching system running stability, friendly interface, the function indexes of the control system can satisfy the requirements of process, has obtained the good application effect.
\end{abstract}

Keywords: Total mixed ration, Batching system, weighing instrument

\section{INTRODUCTION}

Total mixed rations are designed as a homogeneous mixture with the goal to minimize the selective consumption of individual feed components by dairy cattle. It is for this reason that providing feed as a TMR, rather than separately feeding the grain concentrate and forage components, is the most common method of feed delivery used on commercial dairy farms. Total mixed ration feeding technology is that Comprehensive considering the cow growth nutrient requirement of different physiological period and lactation stage and breeding in the process of dairy cattle breeding[12], to design a cow full food recipes by the principle of regulation technology and a variety of feed nutrition collocation according to the formula to all sorts of roughage such as corn, soybean meal, vitamins, minerals and other additives, then using TMR mixer mixing fully get full price of a relatively balanced nutrition diet, so that it can be used for cows feeding technology research by this kind of diet. Compared to the traditional silage and concentrate, green feed such as hay several material feeding of separate from traditional individual breeding method, TMR feeding technology is to avoid cow piddle increase feed intake, improve rumen fermentation, promote digest nutrient of the feed, improve dairy production performance and so on. In other countries, this technology has been popularized to accommodate the development of the production intensification, scale, Mechanization, which achieves good economical benefit in the early 1960s. The technology was recommended in our country in the 1980 s, which the most number of farm was dominated in that time, that the breeding technology has strict requirements for mechanical equipment and formula technology on the farm environment, so it was not until the turn of the century for TMR feeding technology popularization and application.

With the large-scale, intensive transformation of China Dairy Industry, TMR feeding technology aquaculture promotion has been unstoppable. At present, most of the batching system based on PLC [3-4] as the core, to form a complete set of configuration software WinCC as manmachine interface[5-6]. Based on the batching system design as the core, with computer as the upper machine, a company in Changzhou ID510 weighing instrument for the next bit machine, implement the overall control of the system. In the operation process of the upper computer, the system running smoothly, achieving all the requirement of task, the whole system works well, and obtained good effect in practice.

\section{SUMMARY OF BATCHING TECHNOLOGY}

In the batching system, there are a number of coarse material, concentrate, nutrition and water, and other ingredients. The different consist of ingredients can come into different formula, each one of which can feed respectively in the different growth stages and different times. The batching process on the whole can be divided into coarse material into the storehouse, concentrate into warehouse, nutrition and water into the storehouse, warehouse and other major production, involving production equipment has bin, quantitative feeding machine, conveyor and so on. The process shown in Fig-1, the first is coarse 
material transport by conveyor, steering sensor detects when the load almost reached the set value added materials do next, and then the fine material, nutrition, water and other materials and Warehousing when the addition was complete, the delay and start the TMR mixer stirring, mixing, set period of time after the start of the discharge.

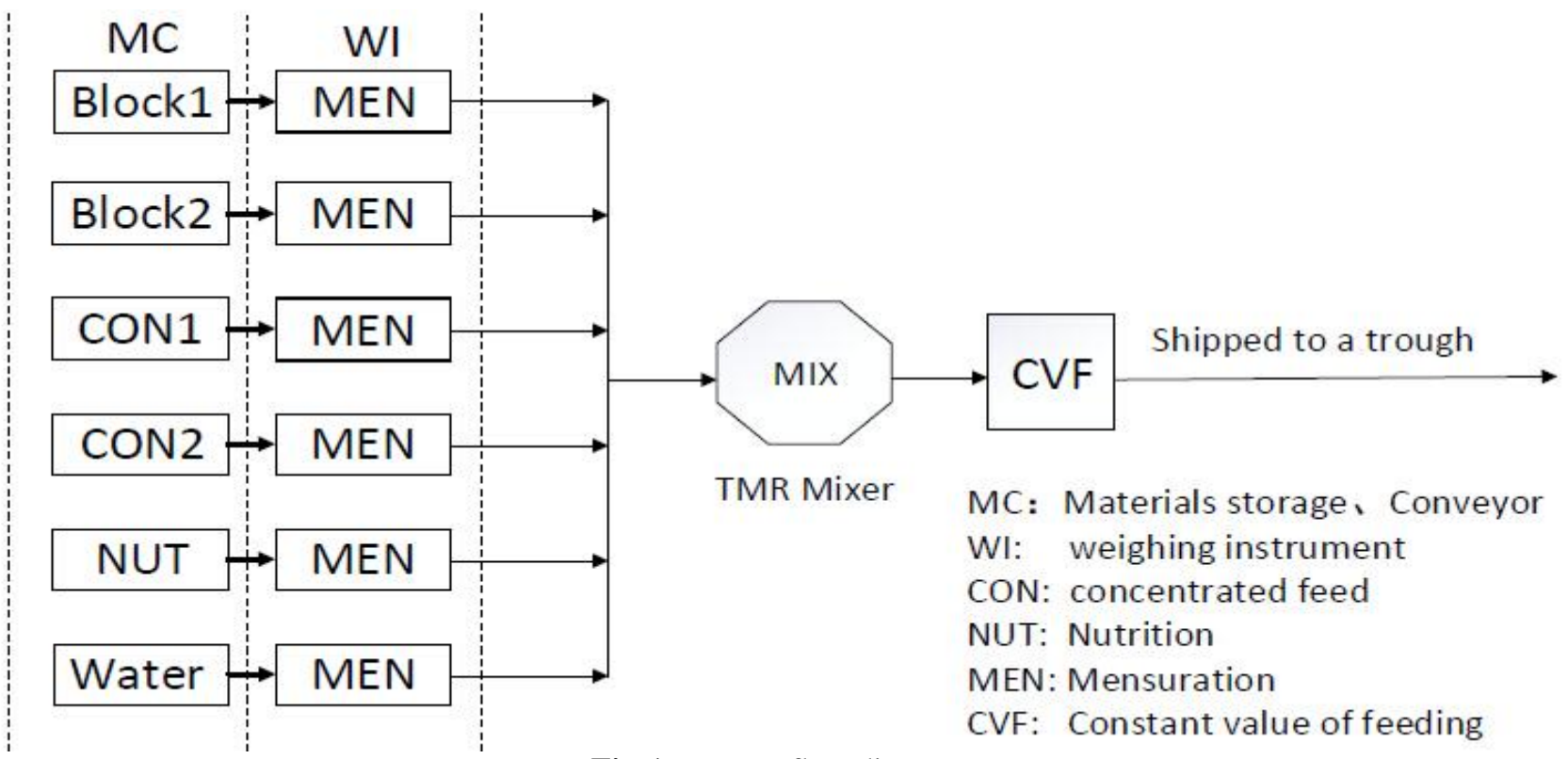

Fig-1: process flow diagram

\section{DESIGN OF THE BATCHING SYSTEM}

\section{OVERALL}

Batching system is mainly composed of TMR mixer, silos, conveyor belt, computer, big screen and the weighing instrument ID510.Divided into mechanical and control two parts, the control part is mainly composed of upper machine software, weighing instrument ID510, big screen, mechanical part is divided into silos, material balance, conveyor belt and TMR mixer, and matches by the weighing sensor and all kinds of motor.

\subsection{Introduction to the Batching System Hardware}

\section{Structure}

Ingredients in the system by Changzhou ID510 weighing instrument in a company as a machine, using $\mathrm{c}++$ Builder 6.0 software writing computer programs, both through RS232 / RS485 serial port communication, complete control of the mechanical parts. ID510 using 12 output interface, control mixer, opening, closing, charging, discharge, water, nutrition, alarming output separately. The system adopts the 16-bit A/D conversion, can fully meet the demand of system accuracy $^{[7]}$. Weighing instrument batching system of a complete set of equipment block diagram is shown in Fig-2:

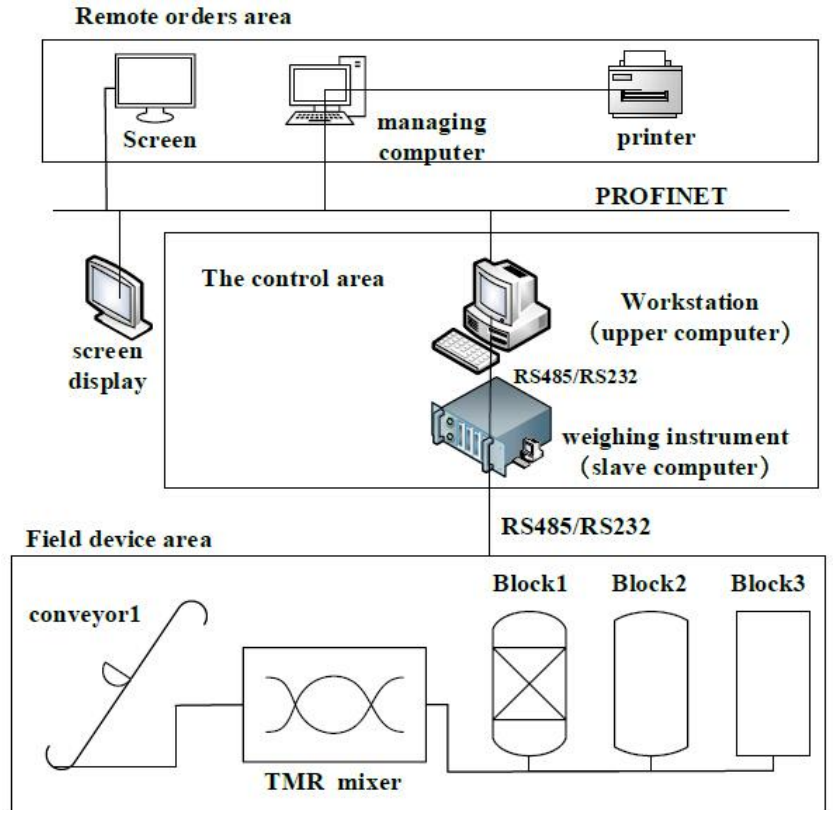

Fig-2: Batching system diagram of the equipment

When the task starts, the remote computer instruction issued production tasks, large screen via Ethernet receive recipe number, while field staff to see the big screen messages, according to the production task recipe table called prior to the recipe storage number, start compounding. Start batching operation, the host computer via the serial port to send information slave machine weighing instrument. when weighing instrument receive the information, at first weighing instrument begin to transfer the information to the site after initialization of automation equipment in accordance with the agreement in advance, control the 
device to start cutting, weighing sensor to weighing of raw materials, slave machine instrument acquisition sensor weighing value. When reaches a certain control frequency converter speed to slow loading, reach a set point to stop cutting, repeat the process until all the raw materials in a single formula, then mixer to start work, stir to open TMR mixer feeding after the prescribed time.

Control part is composed of computer and weighing instrument, the operator first via the requirements of the production tasks, editing in advance in the upper computer production formula, system parameters and production parameters, PC on the one hand, the parameters will be through a database storing the parameters; On the other hand, which transmit the data through a serial port to the slave computer weighing instrument. Weighing instrument store data, calibrated the formula of receiving, then the calibration results back to the upper computer. According to receive the check results, the upper determined whether transmission formula and authorizing start ingredients signal or not, At the same time the slave computer begins to go on when it get the right ingredients. The upper computer return the real-time data by the weighing instrument, on the one hand, it send some ingredients data to the screen display, on the other hand stored the data in the database for later use.

\subsection{Software Design of the Batching System}

This set of batching system can simultaneously support 50 kinds of material and 30 kinds of recipe, to achieve material statistics, database storage, real-time monitoring of materials, report printing and error precision can reach $0.1 \%$. Recipe management mainly includes basic data management, production management, and the control parameters of the production tasks of management. Basic data management including ingredient, recipe composition, etc.; Production control parameter management mainly includes the field equipment needed in the process of control parameter setting and modification; The management of the production tasks that sequential execution of one or more of the following formula. In the ingredient of the monitoring process, it is necessary to display the operation condition of the field devices, and can control field devices, such as startstop, automatic/manual, ensure that operators in the control room can accurately understand the scene of the situation, ensure the smooth progress of the system engineering and to prevent emergency, to ensure the production efficiency, improve the qualification rate of ingredients. The batching system function diagram as shown in List 1

\subsection{Design of the Upper Computer Software}

\subsubsection{Introduction of the Development Tools}

The batching system using $\mathrm{c}++$ builer6.0 as development tool. $\mathrm{C}++$ builder 6.0 is based on Delphi Borland company successful launch of a new generation of development tools, it inherits the Delphi RAD (rapid application development) technology and hundreds of VCL (visual component library). Use the tool controls and Windows API, realize the data communication of the batching system, formula management, database management, report management and printing, etc. The following data Ethernet communication and database management operations

\subsubsection{Serial Communication}

Operator start the ingredient button, PC via a serial port RS232 / RS485 in accordance with the requirements of the production tasks, the production formula (FM_ID), system parameters (PARA_ST) and production (PROC_ID), and other written to the weighing instrument. Weighing instrument received check formula by protocol parsing, the results through a serial port is returned to the upper computer, which to read the returned results determine whether transmit formula and ingredients. At the start of the ingredient, the upper computer read the serial buffer data which is written real-time by the weighing instrument read from the weighing sensor. After the end of the ingredients, start setting the discharge operation, the upper computer is written the data to output buffer, then executed after weighing instruments read correctly.

In order to improve the efficiency of the serial port to read and write operation, using asynchronous overlapping operations. In this operating mode, after the write command, whether or not the write operation is finished, write a function returns immediately. Write operation continues in the background, when the write operation is completed, the notification calling writes thread by a certain way, thus avoiding the primary thread is suspended and improve program efficiency. What's more, On the other hand, the arrival of the data communication time is random, can not be achieved automatically receive an overlapping manner when the data arrives, the event-driven approach to automatically execute the received data, thereby improving the efficiency of program execution.

Serial communication can be written by the Windows API[8], MSCOMM component from Microsoft or other Third company, which will be completed by the Windows API in the article. Batching system using serial communication agreement with slave computer for communication, real-time data transmission, the control for the slave computer weighing instrument. Part of the serial program is as follows:

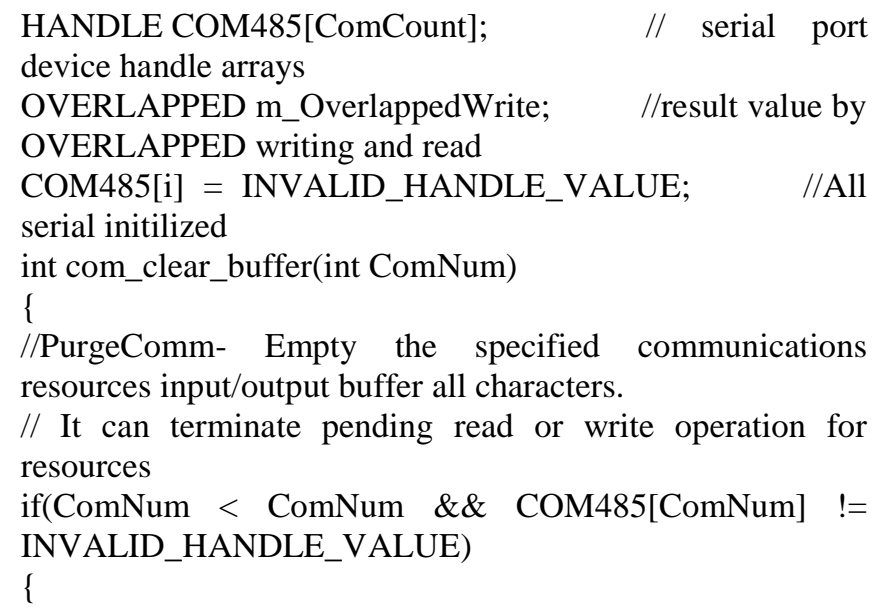


return

PurgeComm(COM485[ComNum],PURGE_TXCLEAR

PURGE_RXCLEAR) ? $1: 0$;

\}

else return 0 ;

\}

//-

void com_init(void)

\{

int $\mathrm{i}$;

for $(\mathrm{i}=0 ; \mathrm{i}<$ ComCount $; \mathrm{i}++)$

\{

COM485[i] = INVALID_HANDLE_VALUE;

\}

//on_exit(COM_free()); // Registered at the end of the called function, release to open the serial port

COM_free();

\}

//-

int com_read( int ComNum, char* pData, int nLength ) // Read write a serial port function

\{

DWORD dwNumRead $=0 ; \quad / /$ A serial port receives the length of the data

ReadFile( COM485[ComNum], pData, (int)nLength, \&dwNumRead, NULL );

return (int)dwNumRead;

\}

//-

int TForm1::TMR_PC_SendCom(char *byte_ptr, int cnt)

\{

DWORD NumWrite; // A serial port data length

if (g_communication_mode_COM_or_LAN==0) \{

WriteFile(COM485[Uart_Port],byte_ptr,

(int)cnt,

\&NumWrite,\&m_OverlappedWrite); //serial send

\}

else \{

NMUDP1->RemoteHost =str510IP;

NMUDP1->SendBuffer(byte_ptr,cnt,cnt); //SEND BY

UDP PROTOCAL

\}

return (int)NumWrite;

\}

//-

m_OverlappedWrite.hEvent $=$ CreateEvent $($ NULL, TRUE, FALSE, NULL ); // Set the initial event to no signal //state FileReadThread = new TFileReadThread(hCommDevice); // //Create a multi-threaded read function

\subsubsection{Database Management}

The role of the database is the data storage, query, management and pass the data to print it. In the process of batching production, essence of the whole control process is to realize the interaction between the data. Data management part of the upper machine mainly includes recipe management, production data query and so on. It is very convenient to satisfy the data management by using database function. Recipe management by using database access technology, implementation of historical formula of modify, add, delete. as well as to the material parameters, advance, tolerance, percentage of changes. Report queries production data refer respectively time node (year, month, day) queries and batch query stage, be achieved by the statistical formula, according to materials summary, ingredients details, discharge and other details.

It is convenient to realize database read and write as well as combining with the remote database by $\mathrm{C}++$ Builder 6.0. This system USES ADO database technology, through ADO and Microsoft ACESS database connection, database written and saved, and other functions. Provide the basic data in the system, database management, storage, operations such as adding and transmitted to print statements. In $\mathrm{c}++$ Builder 6.0 , the database provided by the" TDataSource" control interface, database using SQL operations provided by the "TADOQuery "control method. Part of the function is as follows:

//-

for $(\mathrm{i}=0 ; \mathrm{i}<30 ; \mathrm{i}++)$

\{

mpercent $[\mathrm{i}]=$ StrToFloat $(\mathrm{bfb}[\mathrm{i}]->$ Text $)$;

ADOQuery1->Close();

ADOQuery1->SQL->Clear();

ADOQuery1->SQL->Add("update Recipe_Detail set GET=:p1,GOAL=:p2,ADVANCE=:p3,TOLANCE=:p4,step $=:$ p ,percent=:p6 where repip=:p1 and Material =:p8");

if (g_PEIFANG[THIS_TASK.PeiFang_index].Active[i]==1 )

ADOQuery1->Parameters->Items[0]-> Value = true; else

ADOQuery1->Parameters- $>$ Items[0]-> Value = false;

ADOQuery1->Parameters->Items[1]->Value

g_PEIFANG[THIS_TASK.PeiFang_index].Target[i];

ADOQuery1->Parameters->Items[2]->Value

g_PEIFANG[THIS_TASK.PeiFang_index].Spill[i];

ADOQuery1->Parameters->Items[3]->Value

g_PEIFANG[THIS_TASK.PeiFang_index].Uptol[i];

ADOQuery1->Parameters->Items[4]->Value =

$/ / g \_P E I F A N G\left[T H I S \_T A S K . P e i F a n g \_i n d e x\right] . S t e p[i]$;

ADOQuery1->Parameters->Items[5]->Value = mpercent $[\mathrm{i}]$;

ADOQuery1->Parameters->Items[6]->Value

$=$ StrToInt $($ DBEdit $1->$ Text $) ; / /$ ParamByName

ADOQuery1->Parameters->Items[7]->Value $=\mathrm{i}+1$;

ADOQuery1->ExecSQL(); // EXECUTE

//-

\subsubsection{The Process of the Slave Computer Ingredient}

The slave computer weighing instrument receive the instructions after the upper started the ingredients then send the communication to the equipment which is set. In the 
process of batching, start to delay for a period of time, and then back to zero, the fast charging, when reaches a certain value, slow start charging, when reaches a certain value, start the out-of-tolerance detection, add material operation began after the next test. The batching process as shown in Fig-3.

In the actual operation process, due to environmental constraints, need to start the fixed value feeding function. When the feeding end, stirring evenly mixed, and material and the fixed value feeding hopper can choose emptying two cases. In the working process of the mixer, when hybrid material mixing, sometimes points a few batch of finished goods can be output. when reaches a certain amount of discharge, to stop feeding, so need to set values feeding function.

\subsubsection{Main Interface Design of the Batching System}

Written the upper computer interface by using $\mathrm{c}++$ builder6.0, realized the human-computer interaction, information can be real-time display equipment work, monitor screen, batching process, data report forms printing, etc. Host monitoring interface is most contact, operation command button should try our best to concise and easy to understand. Main interface sample as shown in Fig-4.

This software has realized the PC ingredients monitoring, production tasks, formula management, report management, important parameters, such as help and large screen display switch between interfaces. In the current interface, realize the monitoring of the dispensing equipment, the current state in the water, add water "Shape" control light is green, the water weight is $268.000 \mathrm{~kg}$, mixer total weight is 1634.000 $\mathrm{kg}$, the right side shows the current running state formula, the following green part number to the bullpen, fixed value feeding weight, as well as feeding, feeding, setting start ingredients, stop ingredients such as operation. Left blue frame diagram, for the large screen display content at present. In the diagram below, when check the big screen prompts, can send information to the big screen

List-1 Pasture batching system function

\begin{tabular}{|l|l|}
\hline Recipe Management & batch mixing, edit recipes, downloading recipes \\
\hline $\begin{array}{l}\text { Database } \\
\text { Management }\end{array}$ & $\begin{array}{l}\text { Offers the ingredients of statistical, Material and ingredients statistical query and inquire } \\
\text { History record, Data Storage. }\end{array}$ \\
\hline Communication & $\begin{array}{l}\text { 1.serial communication: between upper computer and instrument by RS485 or RS232 } \\
\text { 2. Ethernet communication: upper computer with managing computer, with screen. }\end{array}$ \\
\hline Alarm & It will provide alarm automatic when system is wrong. \\
\hline Report Printing & Printing statistics according to the database to store information by relevant rules. \\
\hline
\end{tabular}

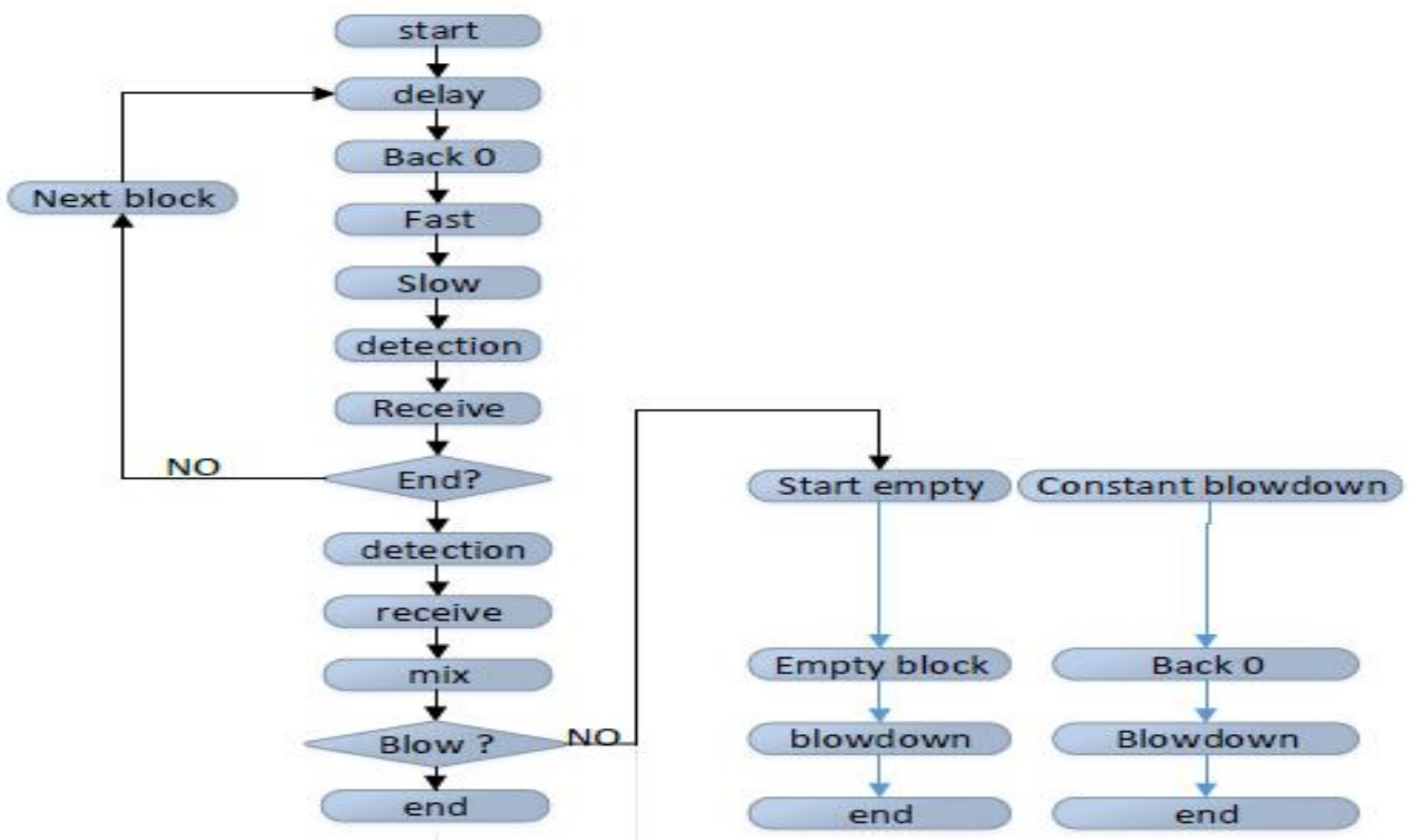

Fig-3: The flow chart of the ingredients 


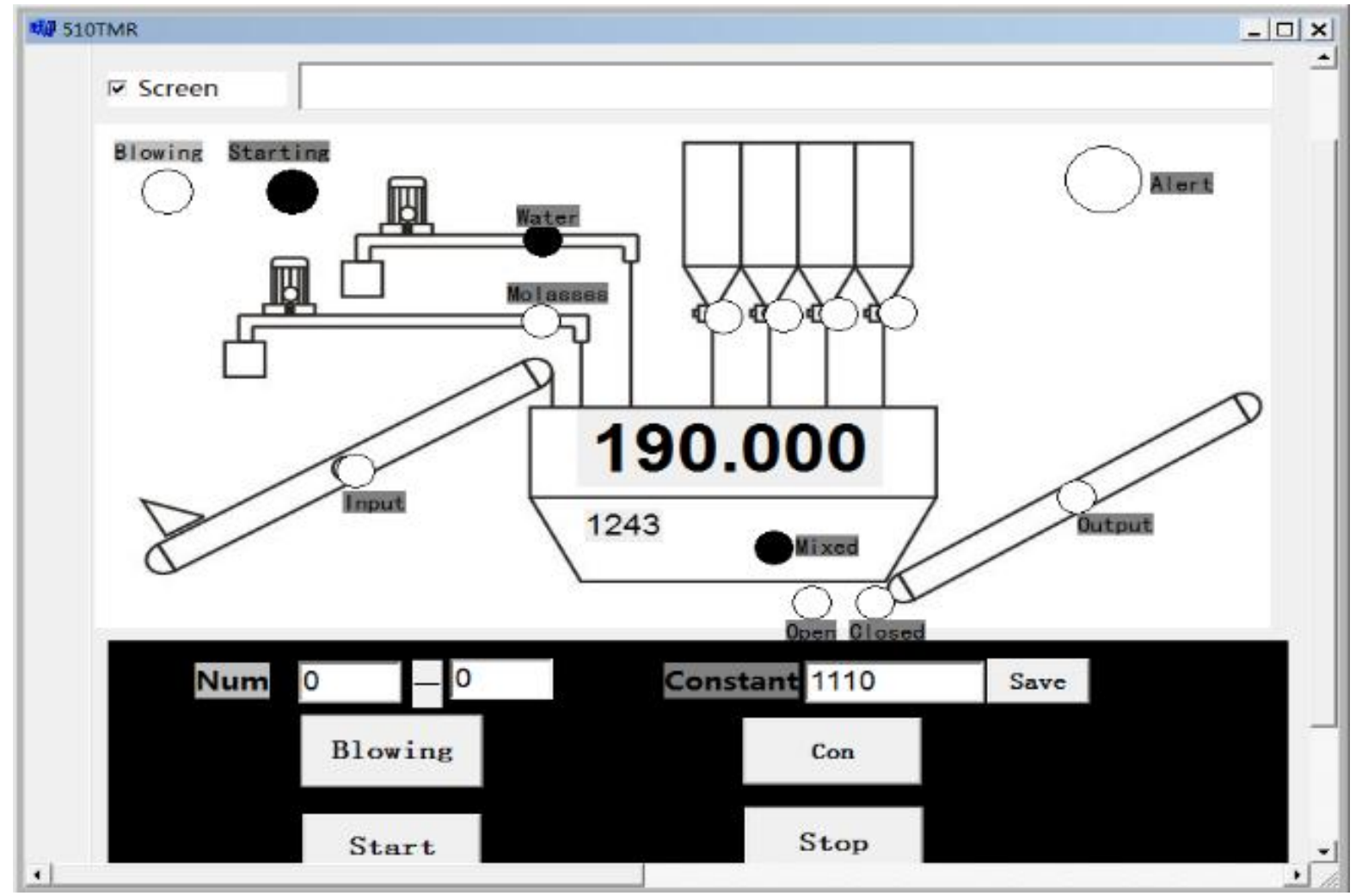

Fig-4: The upper computer interface screenshots

\section{CONCLUSION}

This article describes Design of the modern pasture batching system based on weighing instrument by $\mathrm{C}++$ builder 6.0 , and implemented to achieve a recipe management, use of Access database to achieve the data storage, report printing. Implements the monitoring of the weighing instrument, parameter Settings, simple operation, data accurately. In the field ranch application results show that the system is stable and reliable, simple operation and perfect data record and query system. On the one hand, improve the efficiency of the ingredients and batching accuracy, reduce the material damage to the ingredients, to reach the technological standard; on the other hand, the system to reduce the overall cost of the equipment, the production task can be closed automatically after industrial computer production, greatly reduce the cost of human resources investment, has obtained the good economic efficiency and a broad application prospect.

\section{ACKNOWLEDGEMENTS}

The author would like to thank Professor Zhou who helped and supported with this work. This work was supported by Shanghai University of Engineering Science postgraduate innovation project (14KY0131).

\section{REFERENCES}

[1]. YANG W Z, BEAUCHEMIN K A, RODE L M, et al. Effects of particle size of alfalfa based dairy cow diets on site and extent of digestion [J]. Journal Dairy Science, 2002, 85: 1958-1968.
[2]. EINARSON M S, PLAIZIER J C, WITTENBERG K $M$, et al. Effects of barley silage chop length on productivity and rumen conditions of lactating dairy cows fed a total mixed ration [J]. Journal Dairy Science, 2004, 87:2987-2996.

[3]. Li Wei, Zhu fan, Shao Xinyu. Development and Design of Mixture Monitor System Based on Configuration Software WINCC [J]. Instrument technique and sensor, 2010,04:41-44.

[4]. Zhang Chuanwei, Liu Geng. Research on Silicon Manganese Furnace of Automatic Batching System[J]. Instrument technique and sensor, 2012,10:65-66+69.

[5]. Qiu JianDong, Ou Zhixin. Simulation Analysis of Dynamic Batching System Based on Model Algorithm Control $[\mathrm{J}]$. Computer Measurement \& Control, 2010,09:2095-2097+2100.

[6]. Men Hong, Ying Yuxiang, Wang Zhong. Automatic batching system for cement based on PLC[J]. Manufacturing Automation,2013,01:128-130+136.

[7]. Xu Junlong. Research on One Distributed Automatic Weighting and Batching System [D]. Zhejiang University,2012.

[8]. Nie Cong. Design and Implementation of Configuration Software System Based on Serial Communication[D]. Huazhong University of Science and Technology,2012 


\section{BIOGRAPHIES}

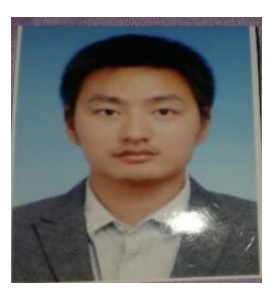

Hu Jie, male, born in 1990.10.7, WuHan

in Hubei province, China, graduate student, mechanical and electrical engineering at the university of Shanghai engineering and technology university, engaged in computer software algorithms.

Zhou Zhifeng, born in December 1976, male, PhD, associate professor, main research based on beidou, GPS intelligent agriculture. 